\title{
A rare case of primary peritoneal hydatid cyst
}

\section{Sanjay Singh*, Mahesh Reddy}

Department of Obstetrics and Gynaecology, Armed Forces Medical College, Pune, Maharashtra, India

Received: 01 November 2016

Accepted: 29 November 2016

\author{
*Correspondence: \\ Dr. Sanjay Singh, \\ E-mail: drsanjaysingh@gmail.com
}

Copyright: (c) the author(s), publisher and licensee Medip Academy. This is an open-access article distributed under the terms of the Creative Commons Attribution Non-Commercial License, which permits unrestricted non-commercial use, distribution, and reproduction in any medium, provided the original work is properly cited.

\begin{abstract}
Primary peritoneal hydatid cyst is a rare entity. A 42 yr old parous female presented with painless lower abdominal lump since 6 months. On clinical examination an immobile abdominopelvic lump of the size of 18-20 weeks pregnant uterus with bosselated surface was noted. Ultrasound Sonography Test (USG) and Contrast Enhanced Computerized Tomography Scan (CECT scan) of abdomen and pelvis revealed large multilocular abdominopelvic cystic mass in continuity with RT ovary. Radiologically a differential diagnosis of hydatid cyst and mucinous cystadenoma was made. All relevant ovarian tumor markers were normal. Serology confirmed increased Echinococcus IgG antibodies (196.60 U/ml, ELISA). The patient underwent complete surgical excision of the lump. Histopathology and Hematoxylin and Eosin ( $\mathrm{H}$ and $\mathrm{E}$ ) staining of the aspirate from the cyst confirmed the diagnosis of hydatid cyst. Since no other organs were involved it was diagnosed as a case of primary peritoneal hydatid cyst. The hydatid cyst may mimic an ovarian cyst, thus it is important to keep this rare entity in mind as a differential in ultrasonographically diagnosed pelvic cystic lesions.
\end{abstract}

Keywords: Echinococcosis, Hydatid cyst, Ovarian cyst, Primary peritoneal hydatidosis

\section{INTRODUCTION}

Human echinococcosis is endemic in Asia, South America, and certain parts of Africa and in some Mediterranean countries. ${ }^{1,2}$ Cystic echinococcosis (Hydatid disease) is a zoonotic and parasitic disease which is caused by the larval form of a tapewormEchinococcus granulosus. The liver is the most commonly affected organ (75\%) followed by lungs $(15 \%) .{ }^{3,4}$ Hydatid Cysts in the peritoneal cavity account for $13 \%$ of the cases ${ }^{1}$ and mainly result from the rupture of concomitant liver cysts-spontaneously or accidentally during surgery. ${ }^{2,5}$ Primary peritoneal echinococcosis however is very rare.$^{6-9} \mathrm{We}$ are presenting a rare case of a primary peritoneal hydatid cyst which was initially confused with an ovarian tumour.

\section{CASE REPORT}

A $42 \mathrm{yr}$ old parous female presented with painless lump lower abdomen since 6 months. It was gradually increasing in size with no gastrointestinal, urinary or menstrual complaints. She also gave history of nausea and minimal loss of weight. There was no significant past medical or surgical history. There was no lymphadenopathy. On per abdominal examination a nontender immobile abdominopelvic lump of the size of 18-20 weeks pregnant uterus was noted. Per vaginal examination revealed a firm mass with bosselated surface felt in the right fornix. A normal size uterus was separately palpable. The differential diagnosis at this point of time was an ovarian tumor.

In view of above the relevant tumor markers (CA125, $\alpha$ Feto protein, b-HCG, Lactate dehydrogenase, Carcinoembryonic antigen and CA 19.9) were done and were found to be normal. Ultrasound Sonography (USG) abdomen and pelvis revealed large multisegmental septate, predominently cystic mass in right (RT) adnexa measuring 14x 9x8 cm (Figure 1). Liver, spleen and other organs showed no cystic lesions. A diagnosis of hydatid cyst with multiple daughter cysts was suggested. Contrast 
Enhanced Computerized Tomography Scan (CECT scan) of abdomen and pelvis showed multilocular cystic mass in right lower abdomen in continuity with right ovary and multiple separate smaller low density cystic masses in peritoneum and mesentery (Figure 2,3). A differential diagnosis of Mucinous cystadenoma with development of peritoneal pseudomyxomas was also considered. On further investigation patient had Eosinophilia (36\%) with absolute Eosinophil count being $3850 / \mathrm{mm}^{3}$. Serology showed increase in Echinococcus IgG Antibody (ELISA) - 196.60 U/ml (Positive > $12 \mathrm{U} / \mathrm{ml}$ ). X-ray chest was normal.

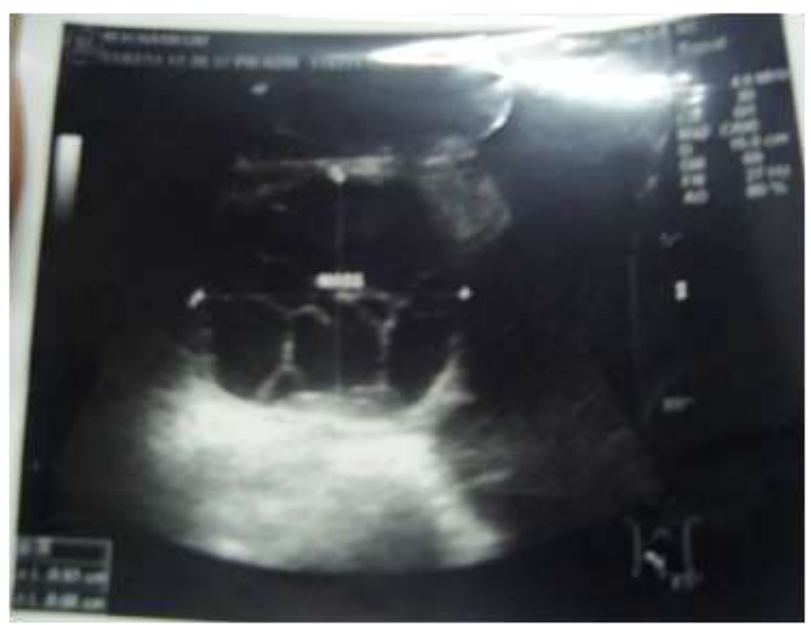

Figure 1: USG abdomen and pelvis showing a welldefined rounded cystic mass with content being of higher echogenicity than clear fluid seen arising from the lower abdomen. Multiple thin septa are noted in it. Image morphology is typical of hydatid cyst.

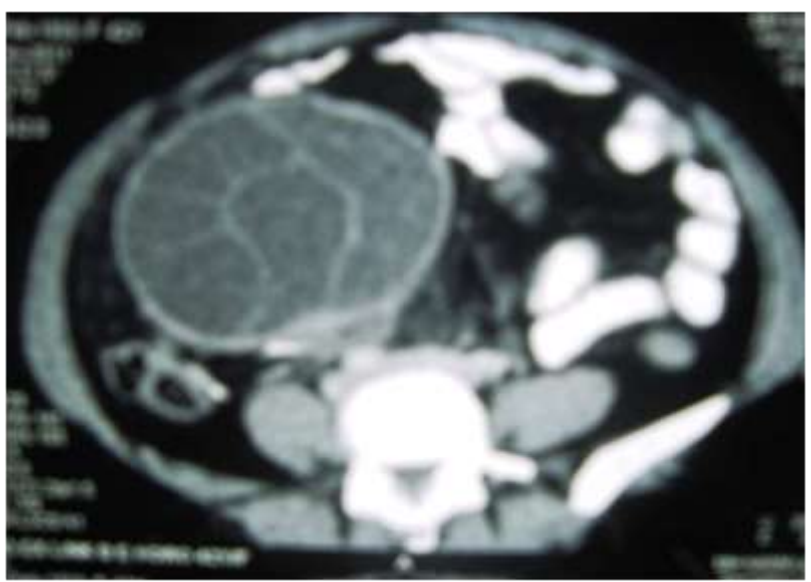

Figure 2: CECT scan of abdomen and pelvis-axial view-showing a fairly large, well defined, thin walled, roughly rounded cystic lesion, in the lower abdomen just to the right of midline. It is anterior to right Psoas. Content of lesion is of high density than clear fluid. Multiple thin septa are seen in it. A small segment of small gut with oral contrast in it is seen just anterior to and in contact with the lesion.

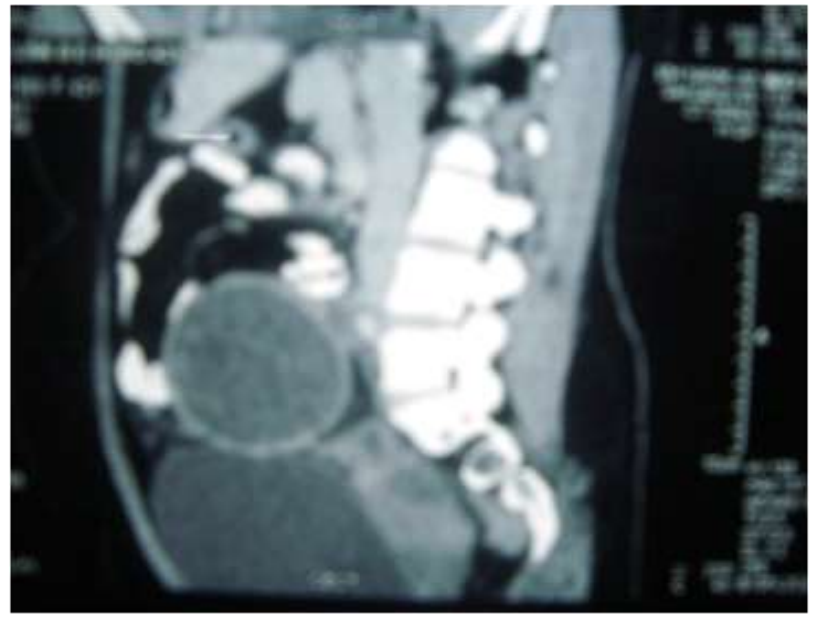

Figure 3: CECT scan of abdomen and pelvis-sagittal reconstruction view- showing inferior aspect of the cystic lesion as described in figure 2 in contact with urinary bladder.

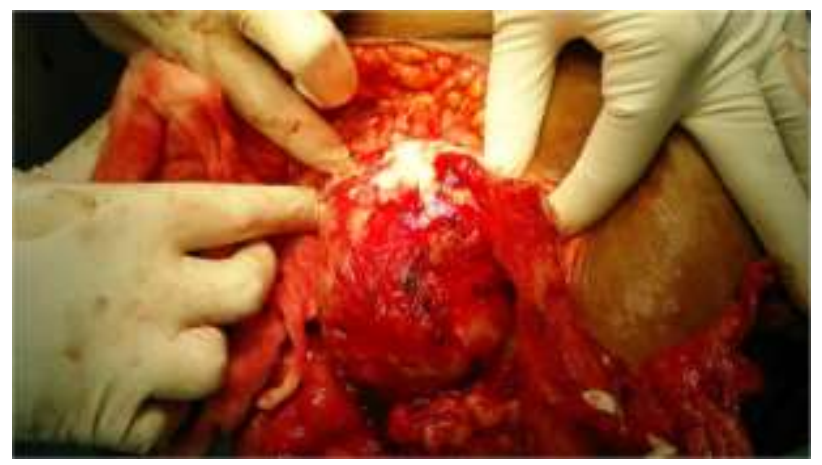

Figure 4: A large cystic mass seen on exploratory laparotomy.

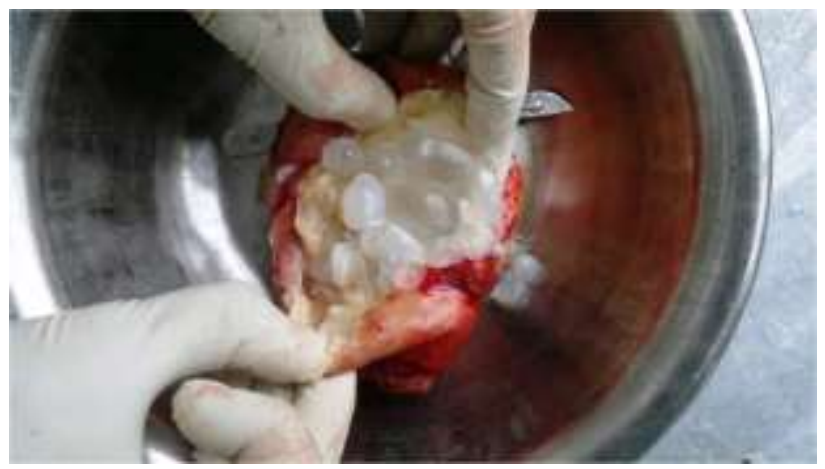

Figure 5: The specimen cut open to show multiple daughter cysts.

There was high index of suspicion of Hydatid cyst based on USG, CECT and positive serology. Ovarian tumor remained a possibility since hydatid cysts are very uncommon in this anatomical location. Patient was put on Tab Albendazole 400mg twice a day (BD) for six weeks. Repeat USG done after six weeks however didn't show any sign of regression. Thus she was taken for exploratory laparotomy. 


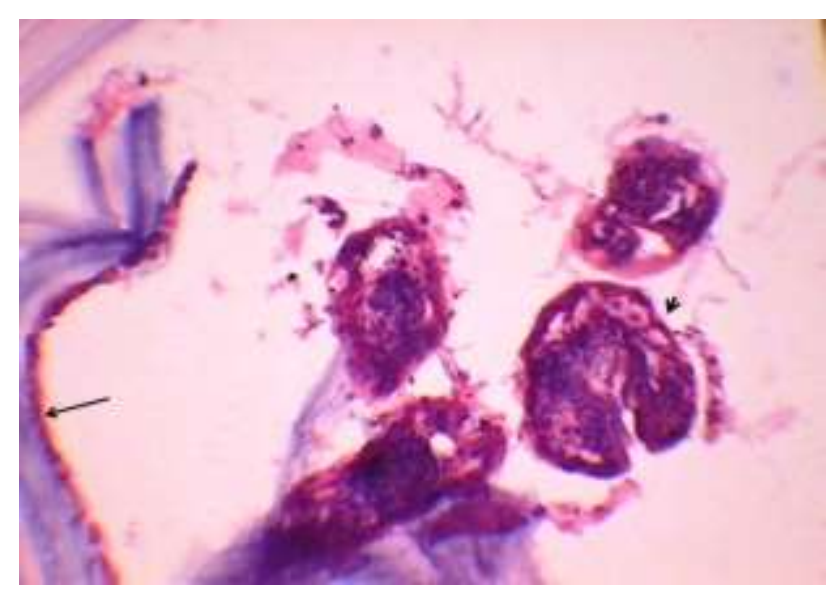

Figure 6: Centrifuged deposit from the aspirate taken from one of the cysts stained with Haematoxylin and Eosin (H and E) stain showed protoscolices. $10 \mathrm{x}$ magnifications.

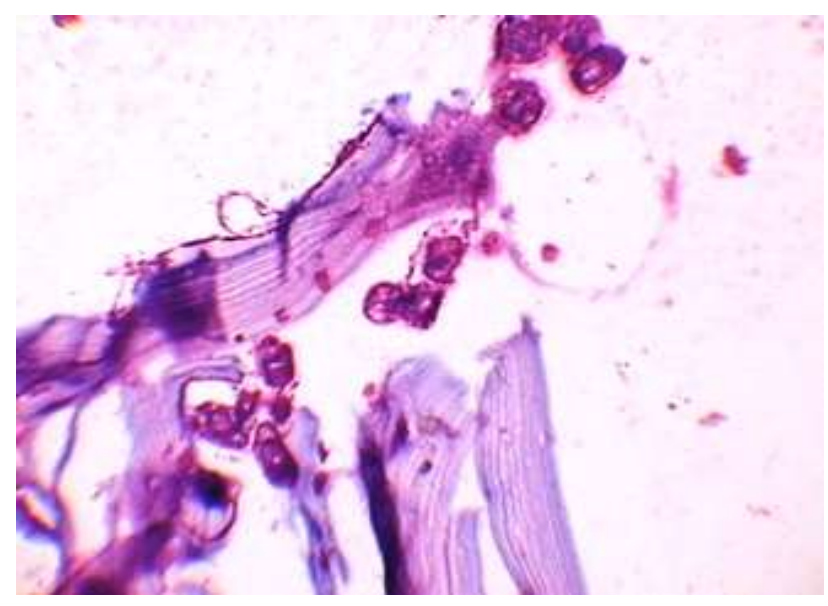

Figure 7: Section from the cyst wall showing thick laminated membrane (Hematoxylin and eosin stain $(H$ and E). 4x magnifications.

On laparotomy a large cystic mass mainly arising from sigmoid mesocolon was found which was very badly adherent to intestines and omentum (Figure 4). Few free cysts were also seen within pelvis. Mass was carefully and completely dissected out. The field of surgery was isolated by OT towel soaked with $3 \%$ hypertonic saline as the scolicidal agent. However the cyst wall never ruptured. Rest of the abdominal organs including liver and spleen didn't show any clinical feature of hydatid cyst. No drain was placed and abdomen was closed in layers. Specimen was sent for histopathological examination (HPE). On grossing, the specimen measured $15 \times 10 \times 8 \mathrm{~cm}$ and on cut section showed multiple small cysts containing clear fluid (Figure 5). The aspirate was taken from one of the cysts and centrifuged deposit stained with Haematoxylin and Eosin ( $\mathrm{H}$ and $\mathrm{E})$ stain showed protoscolices (Figure 6). The biopsy from the specimen further confirmed the diagnosis of hydatid cyst by showing the thick laminated membrane and inner germinal epithelium (Figure 7) and many protoscolices.
Post op recovery was uneventful and patient was continued on Albendazole $400 \mathrm{mg}$ BD for further twelve weeks (four weeks of three cycles with 3 weeks of drug free interval periods). Repeat serology done after completed course of Albendazole turned out to be negative for antibodies. Follow up was planned annually for five years. In her last and final follow up, she was asymptomatic with unremarkable sonogrphic finding of abdomen and pelvis.

\section{DISCUSSION}

Human echinococcosis is a zoonotic and parasitic disease caused by tapeworms of the genus Echinococcus. Out of four the two most important forms of the disease, are cystic echinococcosis (Hydatid disease) caused by Echinococcus granulosus and alveolar echinococcosis caused by Echinococcus multilocularis. Cystic echinococcosis is found in every continent except Antarctica. It is endemic in Asia, South America, certain parts of Africa and in some Mediterranean countries. 1,2

The life cycle of this parasite revolves around two hosts. Carnivores are the definitive hosts and they get infected through the consumption of infected viscera of intermediate host. Various herbivorous act as intermediate hosts and get infected by ingesting the eggs in the carnivores' faeces. The parasitic larval stages develop in their viscera. Humans are accidental intermediate hosts who get infected by ingesting substances infected with Echinococcus eggs. ${ }^{10}$ However they are not able to transmit the disease. Though the cystic echinococcosis is mainly maintained in a dogsheep-dog cycle, several other domestic animals may also be involved, including goats, swine, horses, cattle, camels and yaks.

The hydatid cyst/cysts are located mainly in the liver (75\%) and lungs (15\%), and to a lesser extent in the bones, kidneys, spleen, muscles, central nervous system and eyes. ${ }^{4}$

The incidence of peritoneal involvement is $13 \% .^{1}$ Peritoneal hydatidosis occurs more frequently secondary to hydatid cysts in the liver or rarely in the spleen. It occurs either as a result of seeding from spontaneous ruptures of hepatic cyst or spillage of cyst fluid during surgery. ${ }^{5}$ Both the mechanism can result in anaphylaxis. Primary peritoneal hydatidosis is rare (only $2 \%)^{6-9}$ and dissemination via systemic circulation ${ }^{11}$ or lymphatics ${ }^{12}$ has been postulated as a possible mechanism of occurrence. Since none of the other organs were involved, our case was of primary peritoneal variety. Asymptomatic incubation period may last for many years as was in our case and at times the diagnosis is made when the patient undergoes ultrasonography for an unrelated cause. The signs and symptoms depend on the location, size and pressure exerted by the cyst on surrounding tissues. ${ }^{13}$ Our patient could notice the problem only when the mass was significant in size. The possible differential diagnosis of such intra-abdominal cystic masses may include ovarian cyst, pancreatic cyst, 
mesenteric cyst, lymphangioma, intra-abdominal abscess, loculated ascites etc. ${ }^{14}$ On clinical examination of the patient our first impression was ovarian cyst only. In primary peritoneal hydatidosis, the haemogram may show evidence of eosinophilia, as seen in our case.

USG imaging is the preferred modality for its diagnosis (95\% sensitive). ${ }^{14,15} \mathrm{CE}$ (CT) and/or magnetic resonance imaging (MRI) scans complement or validate the sonogaphy finding. ${ }^{2}$ Various serological tests targeted to detect specific antibodies can support the diagnosis. ${ }^{16,17}$ All these three modalities hinted towards hydatid cyst in our case. USG guided biopsy or aspiration may be performed to differentiate it with tumours or abscess wherever indicated.

Surgery (complete excision) is the treatment of choice for large primary peritoneal hydatid cyst, which was done in this case. Pre-operative courses of Albendazole helps in reducing the number of the cyst, risk of anaphylaxis, tension in the cyst wall (reduces the risk of spillage during surgery) and recurrence rate post-operatively. ${ }^{18,19}$ Intra-operative, use of hypertonic saline or $0.5 \%$ silver nitrate solutions before opening the cyst has been found to kill daughter cysts and therefore prevent further complications as noted above. ${ }^{18,19}$ We gave pre and postoperative Albendazole to our patient and used OT towel soaked with hypertonic saline for the same logic. A recurrence rate of $2 \%$ has been reported in patient undergoing operative intervention. ${ }^{19}$ The efficacy of Albendazole, as sole medical therapy results in successful treatment in up to $40 \%$ of cases. ${ }^{18,19}$

\section{CONCLUSION}

Primary peritoneal hydatid cyst is a rare entity. The patient may remain asymptomatic for longer period of time. It can present as a palpable abdominopelvic lump and one may get confused it with cystic ovarian tumor. Thus, it is important to keep this rare entity in mind as a differential in ultrasonographically diagnosed pelvic cystic lesions. Total surgical excision is treatment of choice and pre and post-operative treatment with Albendazole prevents the recurrence. Long term follow up is prudent to detect and treat any recurrences.

Funding: No funding sources

Conflict of interest: None declared

Ethical approval: Not required

\section{REFERENCES}

1. Karavias DD, Vagianos CE, Kakkos SK, Panagopoulos CM, Androulakis JA. Peritoneal Echinococcosis. World J Surg. 1996;20:337-40.

2. Pedrosa I, Saíz A, Arrazola J, Ferreirós J, Pedrosa CS. Hydatid disease: radiologic and pathologic features and complications. Radiographics. 2000;3:795-817.

3. Tsaroucha AK, Polychonidis AC, Lyrantzopoulos N. Hydatid disease of the abdomen and other locations. World J Surg. 2005;2013:1161-5.

4. Prousalidis J, Tzardinoglou K, Sgouradis L, Katsohis C, Aletras H. Uncommon sites of hydatid disease. World J Surg. 1998;22:17-22.

5. Yuksel M, Demirpolat G, Sever A. Hydatid disease involving some rare locations in the body: a pictorial essay. Korean J Radiol. 2007;2013:531-40.

6. Bannister B, Begg N, Gillespie S. Infectious Diseases; Second Edition, Blackwell Science, UK. 2000;506.

7. Singh RK. A case of disseminated abdominal hydatidosis. J Assoc Physicians India. 2008;2013:55.

8. Khuroo MS. Hydatid disease: current status and recent advances. Ann Saudi Med. 2002;2013:56-64.

9. Balik AA, Celebi F, Basoglu M. Intra abdominal extra hepatic echinococcosis. Surg Today 2001;31:881-4.

10. Chatterjee KD. Parasitology, protozoology and helminthology. 13th edn. New Delhi, India: CBS. 2009;159-65.

11. Aster-cioglu H, Koidor MA, Toplak O. Isolated meso sigmoid hydatid cyst as an unusual cause of colonic obstruction. Report of a case. Surg Today. 2001;31:9202.

12. Iuliano L, Gurgo A, Polettini E. Musculoskeletal and adipose tissue hydatidosis based on the iatrogenic spreading of cystic fluid during surgery: report of a case. Surg Today. 2000;2013:947-9.

13. Parray FQ, Wani SN, Bazaz S. Primary pelvic hydatid cyst: a case report. Case Rep Surg. 2011;2013:809387.

14. Sable S, Mehta J, Yadav S. Primary omental hydatid cyst: a rare entity. Case Rep Surg. 2012;2013:654282.

15. Lewall DB. Hydatid disease: biology, pathology, imaging and classification. Clin Radiol. 1998;53:86374.

16. Sadjjadi SM, Abidi H, Sarkari B. Evaluation of enzyme-linked immunosorbent assay, utilizing native antigen B for serodiagnosis of human hydatidosis. Iran J Immunol. 2007;2013:167-72.

17. Gottstein B, Reichen J. Echinococcosis/Hydatidosis: In: Gordon C, Cook, Alimuddin Zumla, editors. Manson's Tropical Diseases. 21st ed. London: WB Saunders. 2003; 1561-82.

18. Guidelines for treatment of cystic and alveolar echniococcosis in humans. WHO Informal Working Group on Echinococcosis. Bull World Health Organ. 1996;74:231-42.

19. Gourgiotis S, Stratopoulos C: Surgical techniques and treatment for hepatic hydatid cysts. Surg Today. 2007;37:389-95.

Cite this article as: Singh S, Reddy M. A rare case of primary peritoneal hydatid cyst. Int J Reprod Contracept Obstet Gynecol 2017;6:360-3. 